\title{
Motion Compensation for a High Resolution Ka-Band Airborne FM-CW SAR
}

\author{
A. Meta, J. F. M. Lorga, J. J. M. de Wit*, and P. Hoogeboom \\ International Research Centre for Telecommunications-transmission and Radar \\ Delft University of Technology, P. O. Box 5031, 2600 GA Delft, the Netherlands \\ Tel: +31-15-2781034, Fax: +31-15-2784046, Email: a.meta@irctr.tudelft.nl \\ *TNO Defence, Security, and Safety, The Hague, the Netherlands
}

\begin{abstract}
Airborne synthetic aperture radar is a promising new application for FM-CW radars. At the IRCTR, an operational demonstrator system has been developed in order to investigate the practicability of FM-CW SAR and to prove that an FM-CW SAR system can be operated in an efficient manner from a small platform.

The platform used to fly the demonstrator system and the integrated navigation system will be introduced. In addition, the digitalization and the synchronization regarding the navigation system will be addressed. Subsequently, the motion compensation details, especially the squint angle compensation, are explained. The working status of the demonstrator system and the integrated navigation system is proven by the results of an airborne FM-CW SAR campaign. The results obtained during the airborne campaign are presented and the effect of squint angle compensation is shown.

The airborne results could not be further improved by applying more sophisticated motion compensation algorithms or autofocussing techniques. Therefore, it was decided to develop an improved FM-CW front end in order to enhance the FM-CW SAR image quality.
\end{abstract}

\section{INTRODUCTION}

The combination of frequency modulated continuous wave (FM-CW) radar technology and high resolution synthetic aperture radar (SAR) techniques should pave the way for the development of a new generation of small and cost effective imaging radar systems. Airborne SAR is however a very novel application for FM-CW radars. In order to prove the practicability of airborne FM-CW SAR, a fully operational demonstrator system has been developed at the IRCTR, [1] [2]. Moreover, the demonstrator system should prove that an FM-CW SAR system can be operated in an efficient and cost effective manner from a very small platform. Concurrently, special signal processing algorithms, that take into account the characteristics of FM-CW signaling, have been developed, [3]-[4].

In the following section the demonstrator system will be described. Emphasize will be given to the motion sensors and the synchronization of the motion sensors with the radar data acquisition process. In section III, the results obtained during the FM-CW SAR airborne campaign are presented and the improvements after squint angle compensation are shown. Finally, the improved FM-CW front end will be briefly introduced.

\section{The FM-CW SAR DEMONSTRATOR SySTEM}

The demonstrator system operates in stripmap mode. The resolution in range as well as in azimuth direction is around
TABLE I

FM-CW SAR DEMONSTRATOR SYSTEM SPECIFICATIONS (LOW RESOLUTION MODE).

\begin{tabular}{|c|c||c|c|}
\hline Carrier Frequency: & $35 \mathrm{GHz}$ & Antenna Gain: & $24 \mathrm{~dB}$ \\
\hline Frequency Sweep: & $200 \mathrm{MHz}$ & Antenna Isolation: & $52 \mathrm{~dB}$ \\
\hline PRF: & $1000 \mathrm{~Hz}$ & Beamwidth Az./El.: & $6^{\circ} / 28^{\circ}$ \\
\hline Modulation: & Sawtooth & Platform Velocity: & $25 \mathrm{~m} / \mathrm{s}$ \\
\hline IF band: & dc to $2.5 \mathrm{MHz}$ & Altitude: & $150 \mathrm{~m}$ \\
\hline Transmitted Power: & $18 \mathrm{dBm}$ & Max. Range: & $730 \mathrm{~m}$ \\
\hline
\end{tabular}

$1 \mathrm{~m}$. The system specifications are summarized in table I, a detailed system description can be found in [2].

The Stemme S10 light motor glider, manufactured by Stemme GmbH in Germany, provides a relatively cheap test platform. Therefore, it was decided to tailor the FM-CW SAR demonstrator system to this glider. The S10 is a twinseat, high performance, light surveillance glider that can take off unassisted. The maximum endurance time of the S10 is 8 hours, the maximum speed is $61 \mathrm{~m} / \mathrm{s}$, and the maximum altitude is $7.5 \mathrm{~km}$. The glider can be disassembled to fit in a trailer for transportation over the road. Two standardized pods can be mounted under the wings. These pods have a diameter of about $35 \mathrm{~cm}$ and they are $80 \mathrm{~cm}$ long, excluding the aerodynamic fairings that cannot carry any equipment. The maximum payload is $50 \mathrm{~kg}$ per pod. The complete FM-CW SAR demonstrator system has been fitted in such a pod.

The S10 is also the intended platform for the MiniSAR system. MiniSAR is a miniaturized pulse SAR system currently being developed by TNO Defence, Security, and Safety, [5]. The integrated navigation system (INS) under development for MiniSAR, [6], was adapted for use in the FM-CW SAR demonstrator system. In the following subsections, the MiniSAR INS and the synchronization of the INS with the FM-CW SAR data acquisition process will be described in detail.

\section{A. Navigation System Details}

The flexible MiniSAR navigation hardware includes inertial, magnetic, and Global Navigation Satellite System (GNSS) components. The block diagram of the navigation system is shown in Fig. 1.

The inertial component incorporates three accelerometers and three fiber optic gyroscopes. The three Q-Flex accelerometers have temperature sensors inside their housing for a better calibration. This is of high importance due to the fact that the 


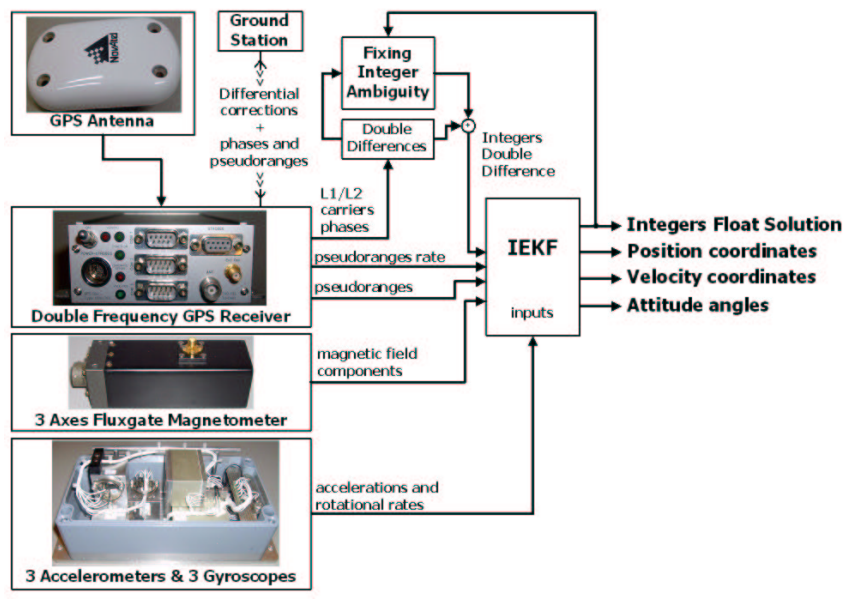

Fig. 1. Flowchart of one of the most advanced working modes of the navigation system.

demonstrator system is installed in the under wing pod, in which the temperature variations may be significant. For the same reason, a temperature sensor is placed next to the fiber optic gyroscopes.

The magnetic component of the system is a three axes fluxgate magnetometer also with a temperature sensor inside its housing. The inertial and magnetic components of the system were calibrated by using the calibration facilities available at the Delft University of Technology. These facilities include a tilt table, a centrifuge, a turn table, and a temperature control chamber, [7]. Also a calibration to the magnetometer was done using the method presented in [8]. This calibration is essential for a good estimation of the navigation system heading angle.

A GPS board with real time kinematics capacity, and the capability of outputting raw pseudoranges, pseudorange rates (carrier Doppler), and carrier phase for both frequencies, forms the GNSS component of the navigation system.

\section{B. Digitalization and Synchronization}

The outputs from the accelerometers, gyroscopes, magnetometer, and temperature sensors are analog signals. These analog signals are acquired with a 16-bit A/D board, which has a maximum sample rate of $100 \mathrm{ksamples} / \mathrm{s}$. The fact that the signals are digitized inside the demonstrator system makes the navigation system more adjustable to different types of platforms and easier to synchronize with the FMCW SAR data acquisition process. The possibility to apply digital filtering when the acquired signals are sampled at a sufficiently high frequency, is another advantage of digitizing the signals inside the demonstrator system.

Synchronization is a primordial issue in any navigation system. Two levels of synchronization have to be taken into account: synchronization of the internal components of the navigation system and synchronization of the navigation system with the FM-CW SAR data acquisition process.

The data acquisition of all motion sensors is triggered by a pulse train with a frequency equal to the sweep repetition frequency. Thus an estimate of the platform position and attitude can be made for each transmitted sweep. The pulse train is synchronized with the radar sweep control signal.

In order to synchronize the GPS data with the remainder of the demonstrator system, strobes from the GPS board were used. The GPS board outputs two strobe signals which make

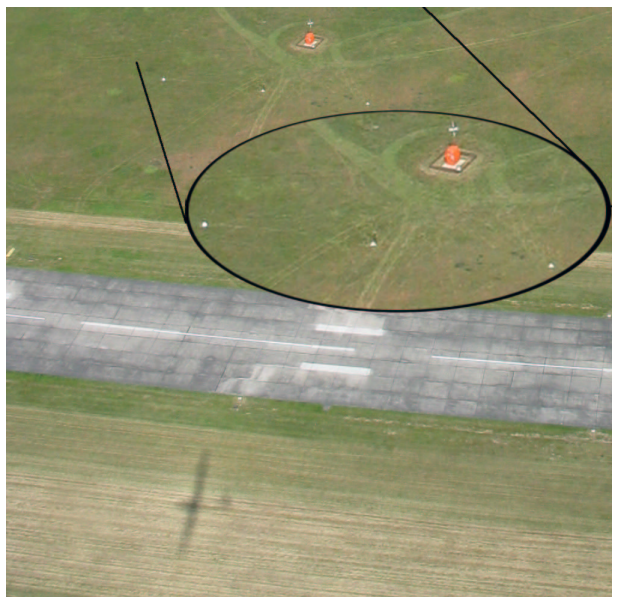

Fig. 2. An airborne picture of the measurement area with a zoom in detail of the radar reflectors. Note the shadow of the glider in the foreground.

the board clock data accessible. The board clock, once it is fine steered to the GPS time, has an accuracy of $\pm 1 \mu \mathrm{s}$. The strobes are a pulse per second signal and a variable frequency signal. Both of these signals are coherent with the GPS data acquired by the board. The final configuration used the GPS variable frequency strobe to produce a pulse train at $1 \mathrm{kHz}$. This pulse train was also digitized by the A/D board. Once the GPS time of the first pulse is known, a GPS time stamp can be given to all samples acquired by the A/D board. The GPS time of the first pulse is obtained by refeeding the pulse train to the GPS board and by making use of the mark input strobe. This strobe registers the time of the received pulse with a resolution better than $55 \mathrm{~ns}$. The registered time is also output by the GPS board and all the information to complete the synchronization process is available.

\section{Airborne Measurements and Motion COMPENSATION}

The airborne campaign was performed at the Strausberg airfield on June $22^{\text {nd }}$ and $23^{\text {rd }} 2004$. Next to the runway, four radar reflectors were placed and their GPS coordinates were measured. Moreover, a GPS ground station was set up in the middle of the measurement scene. An airborne overview of the measurement area is given in Fig. 2.

Several runs were flown along the corner reflectors at 100 , 150 , and $300 \mathrm{~m}$ altitude. Additionally, some flights were made at an altitude of $200 \mathrm{~m}$, during which the engine of the motor glider was switched off. The weather was very turbulent and the wind was directed almost perpendicular to the runway. A squint angle due to the aircraft yaw was therefore present during most measurements.

In SAR processing, the azimuth resolution is obtained by analyzing the Doppler history of the individual target as it is illuminated by the antenna beam. The azimuth resolution is determined by the Doppler bandwidth (in an analogous way, the range resolution is determined by the transmitted bandwidth). An important parameter for the azimuth SAR processing is the Doppler centroid, which depends on angle between the radar and the target. The Doppler centroid $f_{D c}$ is zero if the radar is pointing perpendicular to the flight trajectory; otherwise, if the antenna is squinted, it will be different from zero. It is related to the squint angle by the 
following equation, [9]:

$$
f_{D c}=-\frac{2 v \sin \gamma}{\lambda}
$$

where $v$ is the aircraft velocity and $\lambda$ is the transmitted wavelength. If the Doppler centroid is not corrected, the final image will suffer quality degradation and loss in azimuth resolution. This is due to the fact that the bandwidth of the reference function used for the azimuth compression will not match anymore the target Doppler bandwidth. Using the squint angle information from the navigation system, the reference function is updated every second in order to take in account variation of the Doppler centroid. Squint angle information can also be estimated directly from the radar raw data.

The importance of the squint angle in the motion compensation process can be better understood with the aid of Fig. 3. In the figure, the flight track (the crosses represent the GPS measurements), the position of the radar reflectors, and the location of the GPS ground station are indicated. The radar beam direction shown in red represents the direction of the radar beam without squint angle compensation. The radar beams indicated in green show the beam direction after squint angle compensation. The first airborne FM-CW SAR image is shown in Fig. 4. The responses to the four radar reflectors are visible. The image was produced with data acquired during a flight at $100 \mathrm{~m}$ altitude, the angle of incidence was $65^{\circ}$, and the slant-range to the middle of the scene was around $240 \mathrm{~m}$. In order to enhance the image contrast, 16 azimuth cells were incoherently summed.

In Fig. 5 the effect of the squint angle compensation is shown. The figure shows the azimuth cross section of one of the responses to the radar reflector. The azimuth phase history (before azimuth matched filtering) is shown as well. As can be seen, the response becomes narrower when the data are compensated for the squint angle. The peak level of the response is approximately $5 \mathrm{db}$ higher after squint angle compensation.

\section{FUTURE WORK}

In order to speed up the development and in order to show that an FM-CW SAR can indeed be relatively cheap.

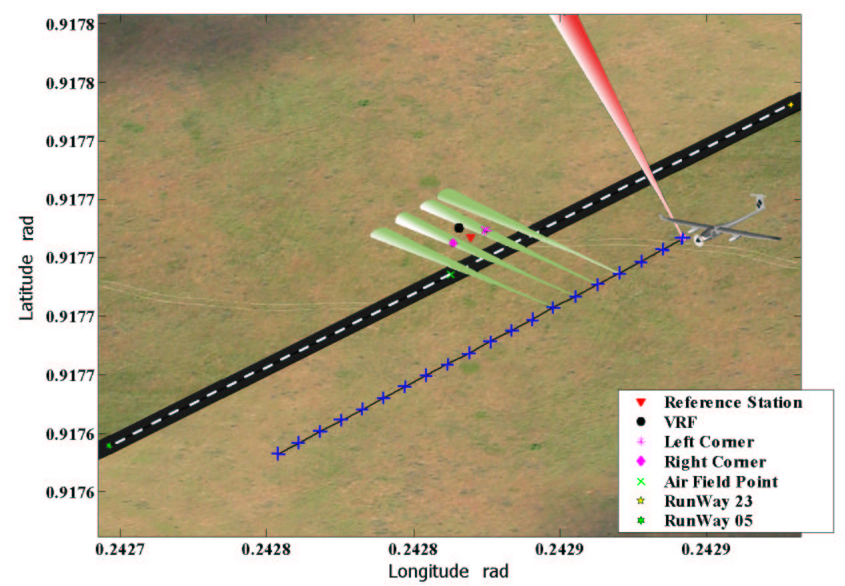

Fig. 3. The flight track, the crosses indicate the GPS measurements. For some GPS points the direction of the radar beam is shown, some corrected for the platform attitude (green) and one uncorrected (red). commercial off-the-shelf components were used as much as possible. As a consequence, the sensitivity of the front end is not sufficient to enable the SAR imaging of areas of low radar reflectivity, such as grasslands, [10]-[11]. Therefore, in Fig. 4 just the responses to the reflectors are visible, details of the background are not observable.

It was expected that the compensation of the along track and cross track motion errors or the application of autofocussing techniques would improve the results. However, this was not the case. Therefore, it was concluded that, in order to obtain a better image quality, the FM-CW front end should be improved. The major difficult with the $35 \mathrm{GHz}$ front end was that it is a black box: the subcomponents are only roughly specified and their output cannot be measured. An in-house development of the RF system gives the advantage that it offers complete control over the system design. Currently a $10 \mathrm{Ghz}$ FM-CW front end is being developed at the TU Delft.

\section{CONCLUSiON}

The main objective of this project was to prove the feasibility of airborne FM-CW SAR under operational circumstances. The practicability of FM-CW SAR was shown by the development of a fully operational airborne demonstrator system. Moreover, the successful airborne campaign proved that an FM-CW SAR system can indeed be operated in an efficient and cost effective manner from a small aircraft. Squint angle correction leaded to an improvement of the SAR images. The Doppler centroid was estimated both using the squint angle information from the navigation system and directly from the radar raw data. In both the cases the results were comparable.

In order to speed up the development, the demonstrator system was based on commercial off-the-shelf components. As a result, the sensitivity of the FM-CW front end does not suffice to image areas of low radar reflectivity. Therefore, it was decided to develop an improved version of the FM-CW front end.

That FM-CW SAR is a very promising concept is further illustrated by the fact that other groups have started working on the subject as well, [12]. Furthermore, the results obtained with the operational MISAR system, [13], prove the usefulness

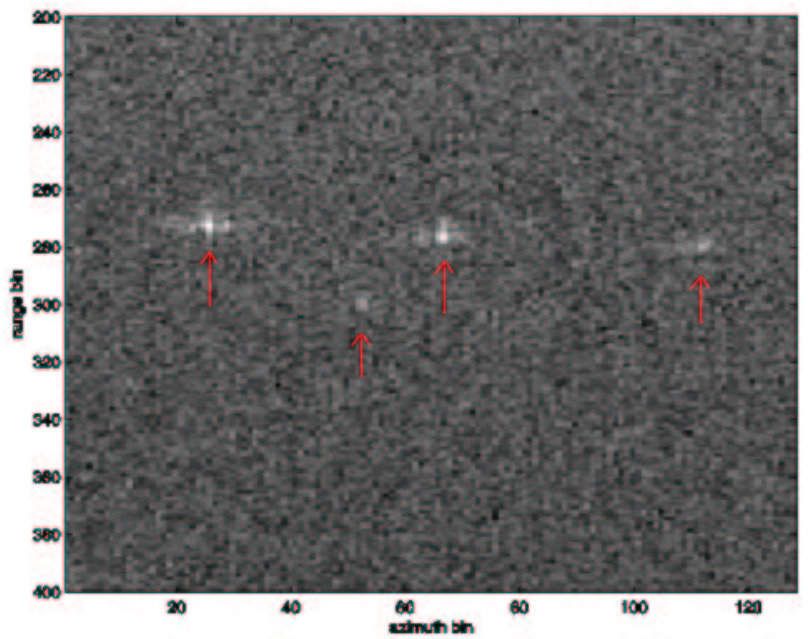

Fig. 4. First Airborne FM-CW SAR image, the responses to the four radar reflectors are indicated by the arrows. 
(a)

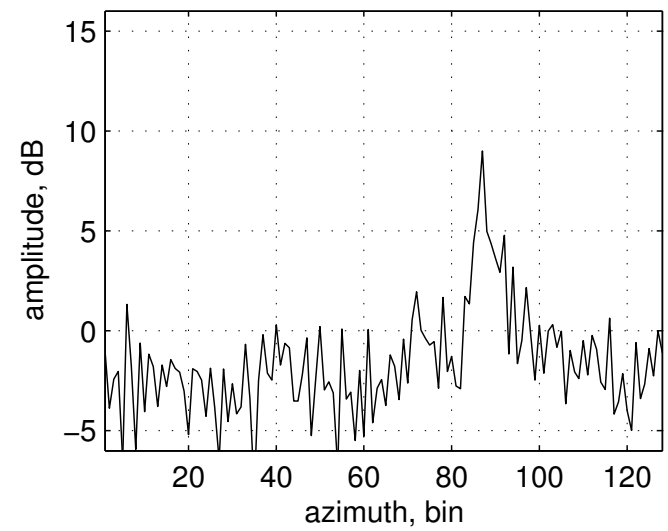

(c)

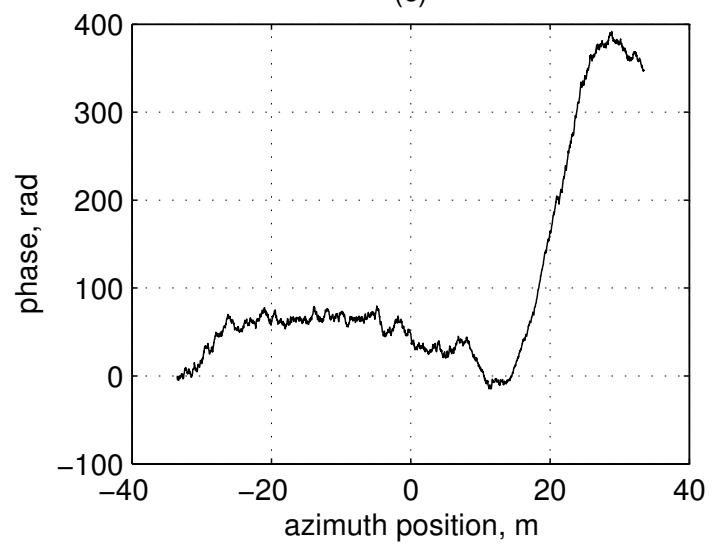

(b)

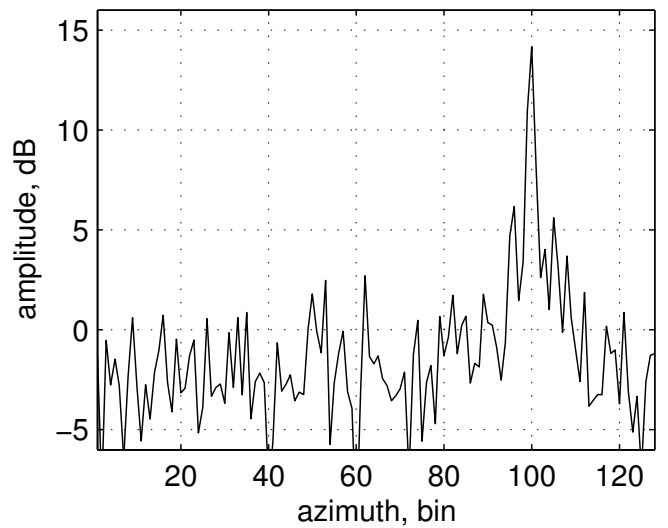

(d)

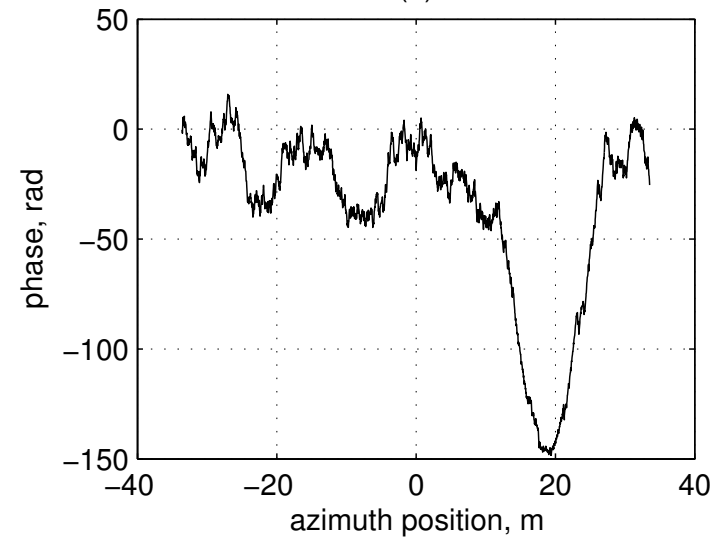

Fig. 5. Azimuth cross section of the response to one of the reflectors; before (a) and after (b) squint angle compensation. The azimuth phase history is shown as well, before (c) and after (d) squint angle compensation.

of an airborne FM-CW SAR system when indeed a dedicated $\mathrm{RF}$ design is made.

\section{ACKNOWLEDGMENT}

The authors would like to thank P. Hakkaart and W. F. van der Zwan of the Delft University of Technology for their contribution to the ground based and airborne experiments.

This work is supported by the Technology Foundation STW, applied science division NWO and the technology program of the Dutch Ministry of Economic Affairs. The project is carried out in close cooperation with TNO Defence, Security, and Safety in The Hague, the Netherlands, and Eonic in Delft, the Netherlands.

\section{REFERENCES}

[1] J. J. M. de Wit, and P. Hoogeboom, and M. P. G. Otten, "Feasibility Study of an FM-CW SAR System," in Proc. CEOS SAR Cal/Val Workshop 2001, Tokyo, Japan, 2-5 Apr., 2001, pp. 119-124.

[2] A. Meta, J. J. M. de Wit, and P. Hoogeboom, "Development of a High Resolution Airborne Millimeter Wave FM-CW SAR," in Proc. EuRAD '04, Amsterdam, the Netherlands, 14-15 Oct., 2004, pp. 209212.

[3] A. Meta and P. Hoogeboom, "High Resolution Airborne FM-CW SAR; Digital Signal Processing Aspects,' in Proc. IEEE IGARSS '03, Toulouse, France, 21-25 July, 2003, vol. VI, pp. 4074-4076.

[4] A. Meta and P. Hoogeboom, "Development of Signal Processing Algorithms for High Resolution Airborne Millimeter Wave FMCW SAR,' in Proc. IEEE Int. Radar Conf. '05, Arlington, U.S.A., 9-12 May, 2005.

[5] P. Steeghs, D. van Halsema, and P. Hoogeboom, "MINISAR: a Miniature, Lightweight, Low Cost, Scalable SAR System," in Proc. CEOS SAR Calibration/Validation Workshop '01, Tokyo, Japan, 2-5 April, 2001, pp. 125-128.
[6] J. F. M. Lorga, W. L. van Rossum, D. van Halsema, Q. P. Chu, and J. A. Mulder, "The Development of a SAR Dedicated Navigation System: From Scratch to the First Test Flight," in Proc. Position Location Navigation Symp. Plans '04, Monterey, California, 26-29 April, 2004, pp. 249-258.

[7] J. F. M. Lorga, Q. P. Chu, and J. A. Mulder, 'Enhancing the Performance of an Integrated Navigation System Using Online and Offline Calibration Procedures," in Proc. European Conf. Aero-Space Sciences, Moscow, Russia, 4-7 July, 2005.

[8] G. H. Elkaim, D. Gebre-Egziabher, J. D. Powell, and W. B. Parkinson, "A Non-Linear, Two-Step Estimation Algorithm for Calibrating SolidState Strapdown Magnetometers," in Proc. $8^{\text {th }}$ IEEE/AIAA St. Petersburg Conf. Navigation Systems, St. Petersburg, Russia, 27-31 May, 2001.

[9] Y. Shao and Z. Zhu, "Squint Mode Airborne SAR Processing using RD Algorithm," in Proc. IEEE Naecon 1997, Dayton, U.S.A., 14-17 July 1997, vol. 2, pp1025-1029.

[10] J. J. M. de Wit, A. Meta, and P. Hoogeboom, "First Airborne FMCW SAR Campaign - Preliminary Results," in Proc. IRS '04, Warsaw, Poland, 19-21 May, 2004, pp. 165-170.

[11] J. J. M. de Wit and P. Hoogeboom, "High Resolution FM-CW SAR Experimental System - First Results,' in Proc. EUSAR '04, Neu-Ulm, Germany, 25-27 May, 2004, vol. 1, pp. 291-294.

[12] M. Nałęcz, K. Kulpa, R. Rytel-Andrianik, S. Plata, and B. Dawidowicz, "Data Recording and Processing in FMCW SAR System," in Proc. IRS '04, Warszawa, Poland, 19-21 May, 2004, pp. 171-175.

[13] M. Edrich, "A High-Resolution Millimeter-Wave FMCW Airborne SAR with Minimum SWP,' in Proc. EUSAR '04, Neu-Ulm, Germany, 2527 May, 2004, vol. 1, pp. 287-290. 